\section{Artículo científico}

Volumen 30(2):469-481. Mayo-agosto, 2019 e-ISSN 2215-3608, doi:10.15517/am.v30i2.33849 https://revistas.ucr.ac.cr/index.php/agromeso/index

\title{
Validación y desarrollo de ecuaciones predictoras de peso para caballos iberoamericanos en Costa Rica ${ }^{1}$
}

\section{Validation and determination of equations prediction weight for Ibero-American horses in Costa Rica}

\author{
Grethel Solano-Mora ${ }^{2}$, Rodolfo WingChing-Jones ${ }^{3}$
}

1 Recepción: 2 de julio, 2018. Aceptación: 17 de octubre, 2018. Este trabajo formó parte de la tesis de Licenciatura en Ingeniería Agronómica con énfasis en Zootecnia de la primera autora, realizada en la Universidad de Costa Rica (UCR), San Pedro, San José, Costa Rica.

2 Criadero Los Tres Potrillos, Aserrí, San José, Costa Rica. gsolano17@outlook.com (autor para correspondencia; https://orcid.org/0000-00025019-9607).

3 Universidad de Costa Rica, Escuela de Zootecnia, Centro de Investigación en Nutrición Animal (CINA), San José, Costa Rica. rodolfo. wingching@ucr.ac.cr (https://orcid.org/0000-0002-8009-2210).

\section{Resumen}

Introducción. Una limitante en los sistemas de producción es el pesaje de los animales, actividad que se realiza de forma empírica o con el uso de cintas de estimación de peso que generan sobre-estimaciones. Objetivo. El objetivo fue validar en caballos iberoamericanos catorce ecuaciones predictoras de peso y desarrollar propias de la raza para Costa Rica. Materiales y métodos. La investigación se realizó entre los meses de septiembre de 2016 y marzo de 2017. Se tomaron las medidas alométricas de peso $(\mathrm{kg})$, longitud $(\mathrm{cm})$, altura a la cruz $(\mathrm{cm})$, perímetro torácico $(\mathrm{cm})$, perímetro umbilical (cm) y edad (meses) de 152 animales (machos y hembras), registrados en la Asociación Centroamericana de Criadores de Caballos de raza iberoamericana. Producto del análisis de la información, se desarrolló una curva de crecimiento para machos $\left(\mathrm{y}=94,54 \ln (\mathrm{X}) 647,14 ; \mathrm{R}^{2}=0,858\right)$ y hembras $(\mathrm{y}=82,09$ $\left.\ln (\mathrm{x})+86,81 ; \mathrm{R}^{2}=0,744\right)$. Resultados. De las ecuaciones evaluadas, se determinó que doce permitieron predecir el peso del animal cuando es un potro $(\mathrm{p}<0,05)$, cinco cuando se considera joven $(\mathrm{p}<0,05)$, seis cuando está en proceso de doma $(p<0,05)$ y seis cuando es adulto $(p<0,05)$. Se determinaron sobre-estimaciones de peso de los animales entre 29,84 y $168,17 \mathrm{~kg}$ de peso vivo, según la ecuación utilizada. De igual manera, las ecuaciones desarrolladas hacen uso de las medidas corporales y edad de los animales, con sobre-estimaciones de peso entre 0,67 y 2,17 kg por animal, lo que permitió tener una mejor predicción del peso y así, mejorar la determinación de los requerimientos nutricionales, dosificaciones de productos veterinarios y el manejo de los animales. Conclusión. Se generaron dos ecuaciones que permitieron estimar el peso del caballo iberoamericano, como también se descartaron las ecuaciones disponibles en la literatura, que no permitieron una estimación del peso según la etapa productiva del animal.

Palabras clave: equinos, pesada, medición del cuerpo, biometría, edad.

\begin{abstract}
Introduction. A limitation in production systems is the weighing of animal, an activity that is performed empirically or with the use of weight estimation tapes which generate overestimates. Objective. The objective was
\end{abstract}


to validate fourteen weigh predictive equations in Ibero-American horses, and develop the breed's own equations for Costa Rica. Material and methods. The investigation was carried out between the months of September 2016 and March 2017. Allometrics measurements of weight $(\mathrm{kg})$, length $(\mathrm{cm})$, height at the withers $(\mathrm{cm})$, thoracic perimeter $(\mathrm{cm})$, umbilical perimeter $(\mathrm{cm})$, and age (months) of 152 animals (male and female) registered in the Asociación Centroamericana de Criadores de Caballos de raza iberoamericana, were taken. As a result on the analysis of the information, a growth curve was developed for males $\left(y=94.54 \ln (X) 647.14 ; R^{2}=0.858\right)$ and females $(y=82.09$ $\left.\ln (\mathrm{x})+86.81 ; \mathrm{R}^{2}=0.744\right)$. Results. From the equations evaluated, it was determined that twelve allowed to predict the weight of the animal when it is a colt $(\mathrm{p}<0.05)$, five when it is considered young $(\mathrm{p}<0.05)$, six when it is in the process of dressage $(\mathrm{p}<0.05)$, and six it when it is adult $(\mathrm{p}<0.05)$.According to the equation used, weight overestimates of animals between $29.84 \mathrm{~kg}$ and $168.17 \mathrm{~kg}$ live weight were determined. Similarly, the developed equations make use of the body measurements and age of the animals, with overestimates of weight between 0.67 and $2.17 \mathrm{~kg}$ per animal, which allowed having a better weight prediction and thus improving the determination of the nutritional requirements, dosages of veterinary products, and the handling of the animals. Conclusion. Two equations were generated to estimate the weight of the Ibero-American horses, as well as discarding the equations available in the literature, which did not allow an estimation of the weight according to the productive stage of the animal.

Keywords: equine, weighing, body measurements, biometry, age.

\section{Introducción}

En Costa Rica, la población de caballos para el año 2014 según el censo agropecuario fue de 66942 animales (INEC, 2015), de los cuales la Asociación Centroamericana de Criadores de Caballos de Raza Iberoamericana (ASOIBERO) maneja en sus registros genealógicos datos de 4590 animales (ASOIBERO, 2016). Los orígenes de esta raza datan desde el año 1860, a partir de las importaciones de animales desde España, los cuales se comienzan a cruzar con caballos criollos y peruanos que se encontraban en el país (Rodríguez, 2016).

En la actualidad, los criadores de caballos iberoamericanos no cuentan con balanza ganadera para estimar el peso de sus animales, parámetro importante para el desarrollo de prácticas de manejo de los semovientes en los criaderos, ya que con él se realiza el cálculo de la dosificación del desparasitante y la alimentación, situación que no es propia de estos sistemas de producción, si no una problemática generalizada en la mayoría de los sistemas que producen animales de interés zootécnico. Como medidas de mitigación de este problema, se buscan opciones para el pesaje de los animales a base de cintas que permiten estimar el peso y, por medio de métodos visuales, que se basan en el uso de medidas alométricas, que se relacionan en una ecuación para predecir el peso y la condición corporal del animal (Cumming, 2009). Esta última alternativa, es la de mayor uso por facilidad, ya que se limita a la toma de medidas del cuerpo del animal, lo que genera una predicción cercana al peso real del mismo (Gibbs y Householder, sf; García et al., 2009). La mayoría de estudios señalan la importancia de registrar tres medidas alométricas: la altura, el perímetro torácico y el largo del animal; esto porque la literatura señala que estas medidas tienen una correlación de 0,79 con el peso del animal, la cual es alta (Carroll y Huntington, 1988; Gamboa, 2012). El inconveniente de las ecuaciones disponibles, es que se desarrollaron en animales con un patrón racial que presentan medidas alométricas similares o que fluctúan en un rango establecido según la edad (Larrea, 2014).

Se encontraron en la literatura catorce ecuaciones disponibles para la estimación del peso de caballos, según la categoría y edad del animal. En Estados Unidos se encontraron diez ecuaciones (Gibbs y Householder, sf; Milner y Hewitt, 1969; Jones et al., 1989; McGowan et al.,2007; Pearson, 2007; Martinson et al., 2014); en España (León, 2007) y Ecuador (Larrea, 2005; Almeida, 2010) se registraron dos por país, mientras que para los países 
de Argentina (García et al., 2009), Australia (McKiernan, 2007) y Costa Rica (Gamboa, 2012), solo se encontró una ecuación de predicción en cada uno. De forma general, las ecuaciones hacen uso de las medidas de perímetro torácico (PT), la altura a la cruz (AC), la longitud (L) y el perímetro umbilical (PU), lecturas que se obtienen en centímetros o pulgadas y expresan el peso del animal en kilogramos o libras, según el país de origen. Situación que provoca diferencias entre el peso estimado con el real.

El objetivo del presente trabajo fue validar en caballos iberoamericanos catorce ecuaciones predictoras de peso y desarrollar propias de la raza para Costa Rica.

\section{Materiales y métodos}

\section{Ubicación y fuente de los datos}

Las valoraciones de los animales se realizaron entre los meses de setiembre de 2016 y marzo de 2017. Este estudio se desarrolló en criaderos ubicados en las provincias de San José, Cartago, Alajuela, Heredia y Puntarenas, Costa Rica. Se tomaron medidas alométricas de 152 caballos iberoamericanos registrados (88 † y $64 \sigma^{\top}$ ), con edades desde los 1,5 meses hasta 172,07 meses.

\section{Ecuaciones de predicción del peso}

Se realizó una búsqueda de información basada en modelos matemáticos para la estimación del peso vivo en caballos. En el Cuadro 1 se resumen las catorce ecuaciones encontradas, las cuales fueron utilizadas en cuatro

Cuadro 1. Ecuaciones para la estimación de peso de los caballos encontradas en la literatura.

Table 1. Equations for weight estimation of horses found in the literature.

\begin{tabular}{|c|c|c|c|c|}
\hline Número & Referencia & País & Tipo de caballo & Ecuación* \\
\hline 1 & Milner y Hewitt (1969) & Estados Unidos & Varios & $\mathrm{PV}(\mathrm{lb})=\left[\mathrm{PT}^{2}\right.$ (inch) $\mathrm{x}$ L(inch) $] / 228,1$ \\
\hline 2 & Carroll y Huntington (1988) & Estados Unidos & Deportivo & $\mathrm{PV}(\mathrm{kg})=\left[\mathrm{PT}^{2}(\mathrm{~cm}) \times \mathrm{L}(\mathrm{cm})\right] / 11900$ \\
\hline 3 & Jones et al. (1989) & Estados Unidos & Varios & $\mathrm{PV}(\mathrm{kg})=\left[\mathrm{PU}^{1,78}(\mathrm{~cm}) \times \mathrm{L}^{0,97}(\mathrm{~cm})\right] 3011$ \\
\hline 4 & McKiernan (2007) & Australia & Varios & $\mathrm{PV}(\mathrm{kg})=\left[\mathrm{PT}^{2}(\mathrm{~cm}) \times \mathrm{L}(\mathrm{cm})\right] / 11000$ \\
\hline 5 & McGowan et al.(2007) & Estados Unidos & Varios & $\mathrm{PV}(\mathrm{kg})=\left[\mathrm{PT}^{2}(\mathrm{~cm}) \times \mathrm{L}(\mathrm{cm})\right] / 11900$ \\
\hline 6 & León (2007) & España & Adultos & $\mathrm{PV}(\mathrm{kg})=4,3 \mathrm{PT}(\mathrm{cm})+3 \mathrm{AC}(\mathrm{cm})-785$ \\
\hline 7 & León (2007) & España & Potros & $\mathrm{PV}(\mathrm{kg})=4,5 \mathrm{PT}(\mathrm{cm})-370$ \\
\hline 8 & Pearson (2007) & Estados Unidos & Varios & $\mathrm{PV}(\mathrm{kg})=\left[\mathrm{PT}^{2}(\mathrm{~cm}) \times \mathrm{L}(\mathrm{cm})\right] / 12000$ \\
\hline 9 & García et al. (2009) & Argentina & Criollo & $\mathrm{PV}(\mathrm{kg})=\left[\mathrm{PT}^{2}(\mathrm{~cm}) \times \mathrm{L}(\mathrm{cm})\right] / 11689$ \\
\hline 10 & Martison et al. (2014) & Estados Unidos & Árabes & $\mathrm{PV}(\mathrm{kg})=\left[\mathrm{PT}^{2}(\mathrm{~cm}) \times \mathrm{L}(\mathrm{cm})\right] / 10996$ \\
\hline 11 & Martison et al. (2014) & Estados Unidos & Pony & $\mathrm{PV}(\mathrm{kg})=\left[\mathrm{PT}^{2}(\mathrm{~cm}) \times \mathrm{L}(\mathrm{cm})\right] / 11069$ \\
\hline 12 & Martison et al. (2014) & Estados Unidos & Trabajo & $\mathrm{PV}(\mathrm{kg})=\left[\mathrm{PT}^{2}(\mathrm{~cm}) \times \mathrm{L}(\mathrm{cm})\right] / 10838$ \\
\hline 13 & García et al. (2009) & Estados Unidos & Deportivo & $\mathrm{PV}(\mathrm{kg})=\left[\mathrm{PT}^{2}(\right.$ inch $) \times \mathrm{L}($ inch $\left.)+22,7\right] / 660$ \\
\hline 14 & Gibbs y Householder (SF) & Estados Unidos & Deportivo & $\mathrm{PV}(\mathrm{kg})=\left[\mathrm{PT}^{2}(\right.$ inch $) \times \mathrm{L}$ (inch) $] / 300$ \\
\hline
\end{tabular}

* PT: perímetro torácico, AC: altura a la Cruz, L: longitud, PU: perímetro umbilical, PV: peso vivo / PT: thoracic perimeter, AC: height at the withers, L: length, PU: umbilical perimeter, PV: liveweight. 
países, donde basan sus cálculos en la recolección de cuatro medidas corporales (PT, AC, L, PU) y están expresadas en dos unidades de longitud ( $\mathrm{cm}$ e in) y de peso ( $\mathrm{kg} \mathrm{y} \mathrm{lb}$ ).

\section{Recolección de datos}

Se tomaron los datos de altura a la cruz $(\mathrm{cm})$, longitud $(\mathrm{cm})$, perímetro torácico $(\mathrm{cm})$, perímetro umbilical $(\mathrm{cm})$, peso $(\mathrm{kg})$ y edad (meses). Para la recolección del peso real de los animales se utilizó una balanza ganadera con capacidad de $3000 \mathrm{~kg}$, un bastón hipómetro para la medición de la altura de caballos y una cinta métrica de $250 \mathrm{~cm}$ para la longitud y el perímetro.

Se tomaron las medidas alométricas sobre una superficie plana, para garantizar que el animal se encontrara bien aplomado, luego se introdujo en una manga de trabajo donde estaba colocada la balanza para el respectivo pesaje. El perímetro torácico se midió al colocar la cinta métrica en la línea del corazón del animal, mientras que, para obtener la medida del perímetro umbilical se colocó la cinta en la línea que pasa por el ombligo del caballo. La altura se tomó, con la ayuda del bastón, del suelo al punto más alto de la cruz, y para la longitud se consideró la distancia desde el húmero (hombro) hasta el isquión.

\section{Desarrollo de una curva de crecimiento}

Con la información recolectada en los sistemas de producción sobre la edad, sexo y el peso real de los animales, se procedió a realizar la curva de crecimiento para machos y hembras del caballo iberoamericano criado en Costa Rica.

\section{Análisis estadístico}

En el caso de las variables perímetro torácico, perímetro umbilical, altura a la cruz, longitud y edad (variables independientes), se realizó un análisis de varianza (ANDEVA) por medio del comando PROC GLM de SAS 9.4 (SAS Institute, 2012), en donde se evaluó el efecto de estas variables sobre el peso de los animales (variable dependiente). Además, se determinó el grado de correlación de estas variables a través del comando PROC CORR de SAS (SAS Institute, 2012). Por último, se realizó un análisis de regresión con la acción Stepwise de SAS/ STAT (SAS Institute, 2012), con la intención de obtener un modelo matemático que permita estimar el peso de los animales según las variables independientes que presentan una significancia menor a $p<0,05$.

Los datos obtenidos de las medidas corporales de los animales muestreados, se sustituyeron en cada ecuación disponible para estimar el peso de los animales. El valor resultante se comparó con el peso real, según lo registrado en la balanza ganadera. Por medio de la acción PROC GLM de SAS (SAS Institute, 2012), se evaluó la significancia de la forma en que se estimó el peso de los animales. Al detectarse diferencias entre medias, se procedió a aplicar la prueba de Duncan $(\mathrm{p}<0,05)$, y así separar las ecuaciones que no presentaron diferencias con el peso real de los animales, de las que sobreestiman o subestiman el peso.

En el caso de las ecuaciones estimadas con el comando Stepwise de SAS (SAS Institute, 2012), estas se validaron en una población nueva, al utilizar una muestra de veinte animales con patrón iberoamericano, que no presentaban registro genealógico, para tal fin se procedió a obtener las medidas de perímetro torácico, perímetro umbilical, altura a la cruz y longitud, como se explicó en el apartado de recolección de datos para cada variable; luego se registró la edad de los animales y se estimó el peso con las ecuaciones desarrolladas. Por medio de un análisis de varianza (ANDEVA), se determinó la capacidad de las ecuaciones desarrolladas en estimar el peso de los animales. 


\section{Resultados}

Los valores promedio de peso, edad y altura a la cruz determinados para el caballo iberoamericano en Costa Rica, por etapa productiva, se observan en el Cuadro 2. Con estos valores se resumen, en el caso de los machos, la ganancia de peso vivo por mes para: pasar de potro a animal joven de $8,46 \mathrm{~kg}$. $\mathrm{mes}^{-1}$, de animal joven a proceso de doma de 3,12 kg.mes ${ }^{-1}$ y de proceso de doma a animal adulto de 0,29 $\mathrm{kg} \mathrm{mes}^{-1}$. En el caso de las hembras, se resumen las ganancias de peso para pasar de una categoría inferior a una superior en 7,74, 2,65 y 0,48 kg.mes ${ }^{-1}$, respectivamente. De igual manera, al observar las Figuras 1 y 2, se nota, en las curvas de crecimiento de machos y hembras de caballos iberoamericanos, respectivamente, un aumento rápido del peso en etapas tempranas de vida con una disminución de la ganancia diaria conforme se llega al peso adulto, según su patrón genético. Lo que concuerda con la correlación entre las medidas fenotípicas de la edad y el peso de los animales, la cual fue positiva y altamente significativa (Cuadro 3).

Cuadro 2. Peso, edad y altura promedio a la cruz obtenidos de la muestra de 152 caballos con fenotipo iberoamericano en Costa Rica, entre setiembre de 2016 y marzo de 2017.

Table 2. Weight, age, and average height at the withers obtained from a sample of 152 horses with Ibero-American phenotype in Costa Rica. Between September 2016 and March 2017.

\begin{tabular}{lccccc}
\hline Clase & $\mathbf{N}^{\circ}$ & Sexo & $\begin{array}{c}\text { Edad promedio por clase } \\
\text { y sexo (meses) }\end{array}$ & $\begin{array}{c}\text { Peso promedio por clase y } \\
\text { sexo (kg) }\end{array}$ & $\begin{array}{c}\text { Altura promedio a la cruz } \\
\text { por clase y sexo (cm) }\end{array}$ \\
\hline Potrillo & 16 & Hembras & $9,95 \pm 4,19$ & $246,56 \pm 59,61$ & $135,47 \pm 6,79$ \\
& 18 & Machos & $7,64 \pm 3,93$ & $212,25 \pm 68,67$ & $130,67 \pm 2,27$ \\
\multirow{2}{*}{ Joven } & 25 & Hembras & $25,86 \pm 5,41$ & $369,78 \pm 44,36$ & $145,66 \pm 3,55$ \\
& 18 & Machos & $26,12 \pm 5,39$ & $368,67 \pm 46,64$ & $148,17 \pm 4,33$ \\
\multirow{2}{*}{ Proceso de Doma } & 11 & Hembras & $46,81 \pm 5,84$ & $425,41 \pm 37,15$ & $150,91 \pm 3,10$ \\
& 14 & Machos & $45,16 \pm 5,78$ & $428,07 \pm 34,26$ & $152,54 \pm 4,08$ \\
Adulto & 36 & Hembras & $99,92 \pm 32,58$ & $450,97 \pm 46,70$ & $150,29 \pm 3,48$ \\
& 14 & Machos & $85,73 \pm 15,69$ & $440,89 \pm 33,95$ & $151,71 \pm 4,21$ \\
\hline
\end{tabular}

Con los datos alométricos de los 152 animales medidos, se obtuvo el peso estimado para cada uno según las ecuaciones descritas en el Cuadro 4. Se determinó que solo las ecuaciones 2, 5, 6, 7, 8 y 9 (Cuadro 4) permitieron hacer una adecuada estimación del peso de caballos iberoamericanos $(\mathrm{p}<0,05)$. Según la información obtenida, las diferencias promedio de peso al utilizar ecuaciones de predicción, fluctuaron entre 1,7 y 168,18 $\mathrm{kg}$ de peso vivo en condiciones de sobreestimación y de $1,43 \mathrm{~kg}$ cuando se subestimó el peso del animal (Cuadro 4).

En el caso de las diferentes etapas de vida que presenta el caballo, como lo son: potros ( 0 a 18 meses), jóvenes (18 a 36 meses), proceso de doma (36 a 60 meses) y adultos (mayores de 60 meses), se dio un comportamiento diferente en la predicción del peso de los animales según la ecuación utilizada (Cuadro 5). En el caso de los potros, doce ecuaciones permitieron estimar un peso que no presentó diferencias significativas con el peso real. Para los animales jóvenes, las ecuaciones se redujeron a cinco, mientras que para animales en proceso de doma y adultos, se podrían utilizar seis ecuaciones para cada categoría.

Para la obtención de los modelos de estimación del peso, y fundamentado en el desconocimiento de la edad de los ejemplares por parte de los dueños de los animales evaluados, se procedió a generar dos ecuaciones de predicción, la primera tomó en consideración solo las medidas corporales (Cuadro 6) y la segunda contempló estas 


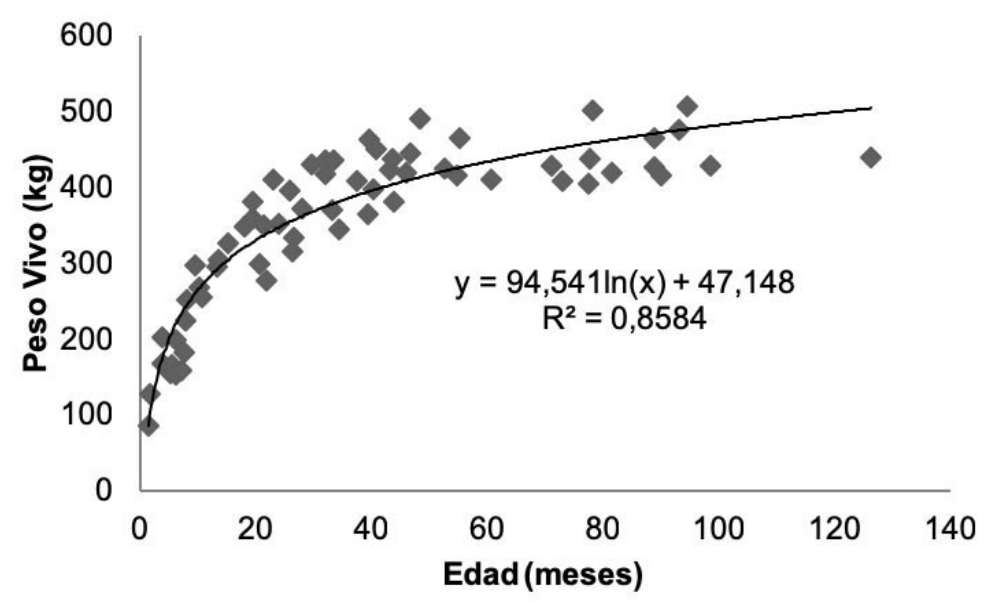

Figura 1. Curva de crecimiento para caballos macho, con fenotipo iberoamericano en Costa Rica. Septiembre de 2016 a marzo de 2017. Figure 1. Growth curve for male horses with an Ibero-American phenotype in Costa Rica. September 2016 to March 2017.

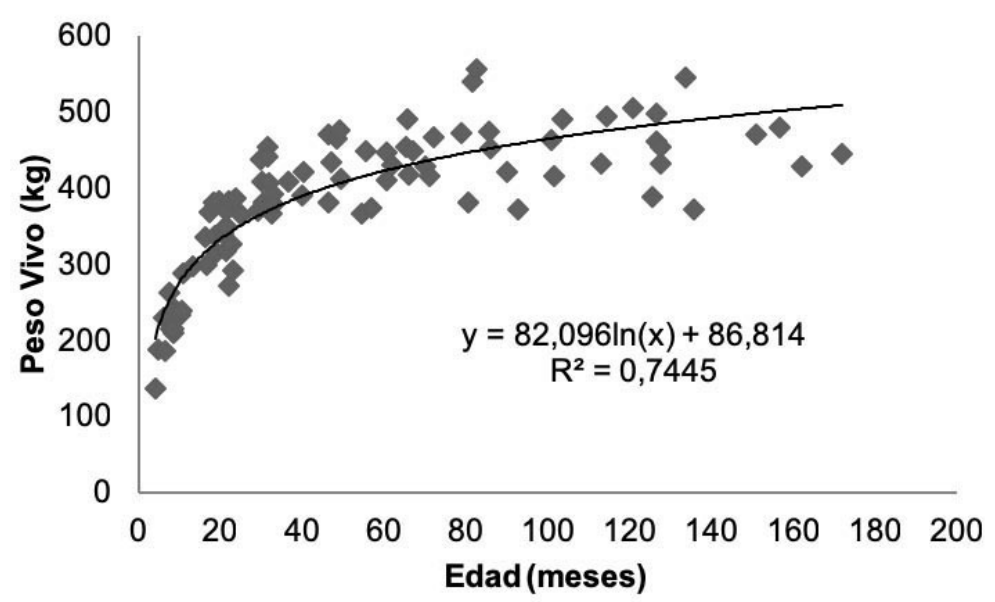

Figura 2. Curva de crecimiento para yeguas, con fenotipo iberoamericano en Costa Rica. Septiembre de 2016 a marzo de 2017.

Figure 2. Growth curve for mares with an Ibero-American phenotype in Costa Rica. September 2016 to March 2017.

e integró la edad de los animales (Cuadro 7). Según la información obtenida, la ecuación de predicción del peso que considera solo las medidas corporales utilizó las variables de perímetro torácico, perímetro umbilical y la longitud; a la vez, descarta la altura a la cruz como variable predictora. Según los resultados, por cada cm de aumento en el perímetro torácico, perímetro umbilical y el largo de los animales, se reflejaría un aumento de 3,03; 0,88 y 1,38 kg de peso vivo, respectivamente. Se resume la ecuación resultante de la siguiente manera: 
Cuadro 3. Coeficientes de correlación de Pearson para la edad, el perímetro torácico, perímetro umbilical, longitud, altura a la cruz y peso de una muestra de 152 caballos con fenotipo iberoamericano en Costa Rica. Setiembre de 2016 a marzo de 2017.

Table 3. Pearson correlation coefficients for age, thoracic perimeter, umbilical perimeter, length, height at the withers, and weight from the sample of 152 horses with Ibero-American phenotype in Costa Rica. September 2016 to March 2017.

\begin{tabular}{|c|c|c|c|c|c|c|}
\hline & Edad & $\begin{array}{c}\text { Perímetro } \\
\text { torácico }\end{array}$ & $\begin{array}{l}\text { Perímetro } \\
\text { umbilical }\end{array}$ & Longitud & $\begin{array}{c}\text { Altura a la } \\
\text { cruz }\end{array}$ & Peso \\
\hline Edad & 1 & $0,6562 *(<, 0001)^{* *}$ & $0,6095(<, 0001)$ & $0,6705(<, 0001)$ & $0,5175(<, 0001)$ & $0,6946(<, 0001)$ \\
\hline Perímetro torácico & & 1 & $0,9229(<, 0001)$ & $0,9236(<, 0001)$ & $0,8961(<, 0001)$ & $0,9754(<, 0001)$ \\
\hline Perímetro umbilical & & & 1 & $0,8362(<, 0001)$ & $0,8337(<, 0001)$ & $0,9235(<, 0001)$ \\
\hline Longitud & & & & 1 & $0,8721(<, 0001)$ & $0,9327(<, 0001)$ \\
\hline Altura a la cruz & & & & & 1 & $0,8920(<, 0001)$ \\
\hline Peso & & & & & & 1 \\
\hline
\end{tabular}

*Coeficiente de correlación, $* *$ Significancia / *Correlation coefficients, ** Significance.

Cuadro 4. Comparación del peso $(\mathrm{kg})$ estimado con el peso real de caballos con fenotipo iberoamericano en Costa Rica y la diferencia promedio, según las ecuaciones de predicción disponibles en la literatura. Setiembre de 2016 a marzo de 2017.

Table 4. Comparison between estimated weight $(\mathrm{kg})$ and the actual weight of horses with an Ibero-American phenotype in Costa Rica and the average difference, according to the prediction equations available in the literature. September 2016 to March 2017.

\begin{tabular}{|c|c|c|c|c|}
\hline & Ecuación & $\mathbf{n}$ & Peso promedio (kg) & $\begin{array}{l}\text { Diferencia de peso } \\
\text { esperada (kg) }\end{array}$ \\
\hline 1 & {$\left[\mathrm{PT}^{2} \mathrm{x}\right.$ L ] $/ 228,1$} & 152 & $541,37^{\mathrm{a}}$ & $168,8 \pm 61,01$ \\
\hline 3 & {$\left[\mathrm{PU}^{1,78} \times \mathrm{L}^{0,97}\right] 3011$} & 152 & $431,85^{\mathrm{b}}$ & $58,66 \pm 36,94$ \\
\hline 13 & {$\left[\mathrm{PT}^{2} \times \mathrm{L}+22,7\right] / 660$} & 152 & $412,52^{\mathrm{bc}}$ & $39,33 \pm 29,11$ \\
\hline 12 & {$\left[\mathrm{PT}^{2} \times \mathrm{L}\right] / 10838$} & 152 & $411,63^{\mathrm{bc}}$ & $38,43 \pm 28,93$ \\
\hline 14 & {$\left[\mathrm{PT}^{2} \times \mathrm{L}\right] / 300$} & 152 & $411,63^{\mathrm{bcd}}$ & $38,43 \pm 28,93$ \\
\hline 10 & {$\left[\mathrm{PT}^{2}\right.$ x L ]/10996 } & 152 & $405,72^{\mathrm{bcd}}$ & $32,52 \pm 27,74$ \\
\hline 4 & {$\left[\mathrm{PT}^{2} \times \mathrm{L}\right] / 11000$} & 152 & $405,57^{\mathrm{bcd}}$ & $32,37 \pm 27,70$ \\
\hline 11 & {$\left[\mathrm{PT}^{2} \times \mathrm{L}\right] / 11069$} & 152 & $403,04^{\text {bcde }}$ & $29,84 \pm 27,21$ \\
\hline 7 & $4,5 \mathrm{PT}-370$ & 152 & $390,20^{\text {cdef }}$ & $17,01 \pm 23,55$ \\
\hline 9 & {$\left[\mathrm{PT}^{2} \times \mathrm{L}\right] / 11689$} & 152 & $381,66^{\text {def }}$ & $8,47 \pm 23,47$ \\
\hline 6 & 4,3PT+3AC-785 & 152 & $378,75^{\text {def }}$ & $5,55 \pm 25,18$ \\
\hline 2 & $\left((\mathrm{PT})^{2} \times \mathrm{L}\right) / 11900$ & 152 & $374,90^{\text {ef }}$ & $1,7 \pm 22,49$ \\
\hline 5 & {$\left[\mathrm{PT}^{2} \times \mathrm{L}\right] / 11900$} & 152 & $374,90^{\text {ef }}$ & $1,7 \pm 22,49$ \\
\hline Real* & 152 & $373,20^{\mathrm{f}}$ & & \\
\hline 8 & {$\left[\mathrm{PT}^{2} \times \mathrm{L}\right] / 12000$} & 152 & $371,77^{\mathrm{f}}$ & $-1,43 \pm 22,08$ \\
\hline
\end{tabular}

PT: perímetro torácico, L: longitud, PU: perímetro umbilical / PT: thoracic perimeter, L: length, PU: umbilical perimeter. *Determinado mediante balanza ganadera / *Determined by means of a livestock balance.

Peso vivo $(\mathrm{kg})=-501,249863+3,032412(\mathrm{PT})+0,877423(\mathrm{PU})+1,384414(\mathrm{~L})$

Donde:

$\mathrm{PT}=$ perímetro torácico $(\mathrm{cm})$.

$\mathrm{PU}=$ perímetro umbilical $(\mathrm{cm})$.

$\mathrm{L}=$ longitud $(\mathrm{cm})$. 
Cuadro 5. Comparación del peso $(\mathrm{kg})$ estimado con el peso real de caballos con fenotipo iberoamericano en Costa Rica, según las ecuaciones de predicción disponibles en la literatura para las diferentes etapas de vida de caballos iberoamericanos. Setiembre 2016 a marzo 2017.

Table 5. Comparison between estimated weight $(\mathrm{kg})$ and the actual weight of horses with an Ibero-American phenotype in Costa Rica, according to the prediction equations available in the literature for the different life stages of Iberoamerican horses. September 2016 to March 2017.

\begin{tabular}{|c|c|c|c|c|c|c|c|c|c|}
\hline \multirow{2}{*}{\multicolumn{2}{|c|}{ Ecuación }} & \multicolumn{2}{|c|}{ Potros } & \multicolumn{2}{|c|}{ Jóvenes } & \multicolumn{2}{|c|}{ Proceso de doma } & \multicolumn{2}{|c|}{ Adultos } \\
\hline & & \multirow{2}{*}{$\frac{\mathbf{N}^{\circ}}{34}$} & \multirow{2}{*}{$\frac{\text { Peso }}{315,34^{\mathrm{a}}}$} & \multirow{2}{*}{$\frac{\mathbf{N}^{\circ}}{43}$} & \multirow{2}{*}{$\begin{array}{c}\text { Peso } \\
536,95^{\mathrm{a}}\end{array}$} & \multirow{2}{*}{$\frac{\mathbf{N}^{\circ}}{26}$} & \multirow{2}{*}{$\frac{\text { Peso }}{609,49^{\mathrm{a}}}$} & \multirow{2}{*}{$\frac{\mathbf{N}^{\circ}}{49}$} & \multirow{2}{*}{$\frac{\text { Peso }}{665,95^{\mathrm{a}}}$} \\
\hline 1 & {$\left[\mathrm{PT}^{2} \mathrm{x} \mathrm{L}\right] / 228,1$} & & & & & & & & \\
\hline 3 & {$\left[\mathrm{PU}^{1,78} \times \mathrm{L}^{0,97}\right] 3011$} & 34 & $271,47^{b}$ & 43 & $429,74^{\mathrm{b}}$ & 26 & $473,37^{\mathrm{b}}$ & 49 & $522,96^{\mathrm{b}}$ \\
\hline 13 & {$\left[\mathrm{PT}^{2} \times \mathrm{L}+22.7\right] / 660$} & 34 & $240,31^{\mathrm{bcd}}$ & 43 & $409,15^{\mathrm{bc}}$ & 26 & $464,42^{\mathrm{bc}}$ & 49 & $507,45^{\text {bc }}$ \\
\hline 12 & {$\left[\mathrm{PT}^{2} \times \mathrm{L}\right] / 10838$} & 34 & $239,77^{\mathrm{bcd}}$ & 43 & $408,27^{\mathrm{bc}}$ & 26 & $463,42^{\mathrm{bc}}$ & 49 & $506,35^{\mathrm{bc}}$ \\
\hline 14 & {$\left[\mathrm{PT}^{2} \times \mathrm{L}\right] / 300$} & 34 & $239,77^{\text {bcd }}$ & 43 & $408,26^{\mathrm{bc}}$ & 26 & $463,41^{\mathrm{bc}}$ & 49 & $506,35^{\text {bc }}$ \\
\hline 10 & {$\left[\mathrm{PT}^{2} \times \mathrm{L}\right] / 10996$} & 34 & $236,33^{\mathrm{bcd}}$ & 43 & $402,4^{\mathrm{cd}}$ & 26 & $456,76^{\mathrm{bcd}}$ & 49 & $499,08^{\mathrm{c}}$ \\
\hline 11 & {$\left[\mathrm{PT}^{2} \times \mathrm{L}\right] / 11069$} & 34 & $234,77^{\mathrm{bcd}}$ & 43 & $399,75^{\mathrm{cd}}$ & 26 & $453,75^{\text {bcde }}$ & 49 & $495,79^{\mathrm{c}}$ \\
\hline 4 & {$\left[\mathrm{PT}^{2} \times \mathrm{L}\right] / 11000$} & 34 & $236,24^{\mathrm{bcd}}$ & 43 & $402,26^{\mathrm{cd}}$ & 26 & $456,59^{\mathrm{bcd}}$ & 49 & $489,9^{c}$ \\
\hline 9 & {$\left[\mathrm{PT}^{2} \times \mathrm{L}\right] / 11689$} & 34 & $222,31^{\mathrm{d}}$ & 43 & $378,54^{\text {def }}$ & 26 & $429,68^{\mathrm{def}}$ & 49 & $469,49^{d}$ \\
\hline 2 & $\left((\mathrm{PT})^{2} \times \mathrm{L}\right) / 11900$ & 34 & $218,37^{\mathrm{d}}$ & 43 & $371,83^{\mathrm{ef}}$ & 26 & $422,06^{\mathrm{f}}$ & 49 & $461,17^{\mathrm{d}}$ \\
\hline 5 & {$\left[\mathrm{PT}^{2} \times \mathrm{L}\right] / 11900$} & 34 & $218,37^{\mathrm{d}}$ & 43 & $371,83^{\mathrm{ef}}$ & 26 & $422,06^{\mathrm{f}}$ & 49 & $461,17^{\mathrm{d}}$ \\
\hline 8 & {$\left[\mathrm{PT}^{2} \times \mathrm{L}\right] / 12000$} & 34 & $216,55^{\mathrm{d}}$ & 43 & $368,73^{\mathrm{f}}$ & 26 & $418,54^{\mathrm{f}}$ & 49 & $457,32^{\mathrm{d}}$ \\
\hline 6 & 4,3PT+3AC-785 & 34 & $219,19^{d}$ & 43 & $386,38^{\text {cdef }}$ & 26 & $435,32^{\text {cdef }}$ & 49 & $452,74^{\mathrm{d}}$ \\
\hline \multirow[t]{2}{*}{7} & $4,5 \mathrm{PT}-370$ & 34 & $263,57^{\mathrm{bc}}$ & 43 & $395,26^{\text {cde }}$ & 26 & $431,26^{\text {def }}$ & 49 & $451,85^{\mathrm{d}}$ \\
\hline & Peso Real & 34 & $228,4^{\mathrm{cd}}$ & 43 & $369,31^{\mathrm{f}}$ & 26 & $424,85^{\text {ef }}$ & 49 & $449,67^{\mathrm{d}}$ \\
\hline
\end{tabular}

PT: perímetro torácico, AC: altura a la cruz, L: longitud, PU: perímetro umbilical / PT: thoracic perimeter, AC: height at the withers, L: length, PU: umbilical perimeter.

En la segunda ecuación (Cuadro 8), la cual utiliza las cinco variables consideradas en el estudio, al igual que con la ecuación anterior, se nota, que por cada cm de aumento en el perímetro torácico, el animal presentó un aumento en peso cercano a los $3 \mathrm{~kg}$, en este caso, se valoró $2,75 \mathrm{~kg}$. A continuación se resume la ecuación obtenida:

Peso vivo $(\mathrm{kg})=-514,33411+0,20702(\mathrm{E})+2,74792(\mathrm{PT})+0,81847(\mathrm{PU})+1,02748(\mathrm{~L})+0,79159(\mathrm{AC})$

Donde:

PT: perímetro torácico $(\mathrm{cm})$.

PU: perímetro umbilical $(\mathrm{cm})$.

L: longitud $(\mathrm{cm})$.

AC: altura a la cruz $(\mathrm{cm})$.

E: edad (meses)

Como parte de los resultados, los modelos de predicción obtenidos se validaron en una población de veinte animales que no presentaban registro genealógico, con edades entre 5 a 180 meses. No se obtuvieron diferencias significativas $(\mathrm{p}>0,05)$, debido a que las diferencias del peso real de los animales tomado con balanza ganadera 
Cuadro 6. Ecuación de predicción del peso de caballos con fenotipo iberoamericano en Costa Rica, asociados a las medidas corporales. Setiembre 2016 a marzo 2017.

Table 6. Weight prediction equations of horses with Ibero-American phenotype in Costa Rica, associated with body measurements. September 2016 to March 2017.

\begin{tabular}{lccccc}
\hline Variable & $\begin{array}{c}\text { Estimador de } \\
\text { parámetro }\end{array}$ & Error estándar & Tipo II SS & F-Valor & Pr>F \\
\hline Intercepto & $-501,249863$ & 14,285354 & 432506 & 1231,19 & $<, 0001$ \\
Perímetro torácico & 3,032412 & 0,303631 & 35039 & 99,74 & $<, 0001$ \\
Perímetro umbilical & 0,877423 & 0,197244 & 6951,45 & 19,79 & $<, 0001$ \\
Longitud & 1,384414 & 0,241661 & 11529 & 32,82 & $<, 0001$ \\
\hline
\end{tabular}

Cuadro 7. Ecuación de predicción del peso de caballos con fenotipo iberoamericano en Costa Rica, asociados a las medidas corporales y edad. Setiembre 2016 y marzo 2017.

Table 7. Weight prediction equations of horses with Ibero-American phenotype in Costa Rica, associated with body measurements and age. September 2016 and March 2017.

\begin{tabular}{lccccc}
\hline Variable & $\begin{array}{c}\text { Estimador de } \\
\text { parámetro }\end{array}$ & Error estándar & Tipo II SS & F-Valor & Pr>F \\
\hline Intercepto & $-514,33411$ & 28,86725 & 101643 & 317,45 & $<, 0001$ \\
Edad & 0,20702 & 0,05265 & 4949,65 & 15,46 & 0,0001 \\
Perímetro torácico & 2,74792 & 0,30961 & 25221 & 78,77 & $<, 0001$ \\
Perímetro umbilical & 0,81847 & 0,18915 & 5995,33 & 18,72 & $<, 0001$ \\
Longitud & 1,02748 & 0,25094 & 5367,89 & 16,77 & $<, 0001$ \\
Altura a la cruz & 0,79159 & 0,38502 & 1353,43 & 4,23 & 0,0416 \\
\hline
\end{tabular}

Cuadro 8. Validación de las ecuaciones desarrolladas para estimar el peso de caballos con fenotipo iberoamericano en Costa Rica. Septiembre 2016 a marzo 2017.

Table 8. Validation of the equations developed to estimate the weight of horses with an Ibero-American phenotype in Costa Rica. September 2016 to March 2017.

\begin{tabular}{lcc}
\hline Ecuaciones & n & Peso $(\mathbf{k g})^{*}$ \\
\hline$-501,249863+3,032412(\mathrm{PT})+0,877423(\mathrm{PU})+1,384414(\mathrm{~L})$ & 20 & 334,42 \\
$-514,33411+0,20702(\mathrm{E})+2,74792(\mathrm{PT})+0,81847(\mathrm{PU})+1,02748(\mathrm{~L})+0,79159(\mathrm{AC})$ & 20 & 335,97 \\
Peso real & 20 & 333,80 \\
\hline
\end{tabular}

$*(\mathrm{p}>0,05) / *(\mathrm{p}>0.05)$.

PT: perímetro torácico, AC: altura a la cruz, L: longitud, PU: perímetro umbilical, E: edad / PT: thoracic perimeter, AC: height at the withers, L: length, PU: umbilical perimeter, E: age.

al calculado con la ecuación, fue de $0,62 \mathrm{~kg}$ cuando solo se usaron variables alométricas y, de 2,17 kg cuando se consideró la edad y la altura a la cruz. 


\section{Discusión}

El patrón racial del caballo iberoamericano señala que, a partir de los tres años, los machos deben alcanzar una altura mínima de 1,50 m y las hembras $1,48 \mathrm{~m}$, y que de 1,52 y 1,50 m son las medidas ideales para machos y hembras, respectivamente (ASOIBERO, 2013). Se observó que los animales a partir del proceso de doma, momento en que alcanzan los tres años, registraron una altura a la cruz mínima permitida. Lo anterior indica que las estrategias de selección que presentan los criaderos cumplen con el estándar de la altura en los animales, lo cual permitirá mejorar el fenotipo del caballo iberoamericano. Estrategia que se apoya en las curvas de crecimiento de los animales, la cual permite, según su etapa de desarrollo, asociar en los semovientes procesos de sobre o subalimentación según la edad y el peso (Gandarillas, 2008). Si los animales presentaran variaciones en el peso según la edad con la curva ideal de crecimiento, podrían estar predispuestos a desórdenes ortopédicos (Gandarillas, 2008). Asimismo, con estas curvas se puede observar en qué momento los animales alcanzan su peso adulto, lo que ayuda a conocer el manejo alimenticio que se les debe dar a los equinos. Se puede alimentar los caballos para que alcancen los pesos deseados más rápido, sin embargo, esto les puede generar afecciones ortopédicas (Gibbs y Potter, sf; Gandarillas, 2008).

De las catorce ecuaciones para la estimación del peso de los caballos, solo el 42,86 \% de la información disponible en la literatura, se podría utilizar en este tipo de animal. Con las diferencias determinadas se pueden generar problemas en la dosificación de medicamentos, desparasitantes y los aportes de nutrimentos, debido a que se sobreestiman los requerimientos, lo que genera problemas de salud en los animales (Gibbs y Householder, sf; García et al., 2009). En el caso del comportamiento observado entre las ecuaciones de estimación de peso y las etapas de vida de los animales en este trabajo, se determinó que a menor edad del animal, se obtuvieron más (doce) ecuaciones que permiten estimar el peso de los animales, lo cual podría estar explicado por las razas que dan origen a este animal, como los caballos de razas peruanas, pura raza española, costarricense de paso y lusitano (ASOIBERO, 2005), donde se presentan razas en común (Librado et al., 2016). También podría asociarse a la alimentación de los caballos en sus primeros años, donde el factor ambiental no genera tanto efecto (leche de la yegua, alimento balanceado y forraje), como en etapas de mayor edad, donde se valora la composición y la cantidad de los insumos alimenticios ofrecidos (forrajes frescos, henos y alimentos balanceados), y las condiciones de manejo de los animales (LaVigne et al., 2015).

El desarrollo de modelos que permitan estimar el peso de animales con datos propios, concuerda con lo señalado por Takaendegan et al. (2012), quienes mencionaron que un modelo para la predicción del peso vivo en caballos es más exacto cuando se incluyen variables independientes en el mismo. En este caso al tomar en cuenta la edad, y la altura a la cruz, da como resultado la incorporación de dos factores importantes para la determinación del peso, aunque sean las variables con menor correlación con el peso de los animales (edad $=0,69455$, y altura a la cruz $=0,89203$ ). En el caso de la relación de la edad de los animales y su peso, largo, altura a la cruz, perímetro torácico y umbilical, las diferencias se podrían asociar al cruce del cual procede el animal, donde Solano y WingChing-Jones (2018) informaron de siete cruces para la obtención de un animal con patrón iberoamericano, donde el macho reproductor, puede ser un caballo de la raza pura española (tres cruces), costarricense de paso (dos cruces) o iberoamericano con influencia española o costarricense de paso (dos cruces), y el cruce de preferencia es una hembra costarricense de paso con un macho de pura raza española.

Las ecuaciones desarrolladas en este trabajo permitieron una mejor estimación del peso de los animales que las ecuaciones disponibles en la literatura. Tal comportamiento se sustenta en la determinación de altas correlaciones entre variables fenotípicas $(\mathrm{p}<0,001)$ y moderadas con la edad de los animales, según los rangos que señalan Nieves y Domínguez (2009), donde consideraron correlaciones con altos valores entre 0,8 a 1 y correlaciones moderadas de 0,5 a 0,8 . En el caso de la correlación entre la altura a la cruz y el peso, esta fue de 0,89 , valor mayor al informado por Carroll y Huntington (1988) de 0,62 en caballos de la raza Thorougbred, caracterizados por su agilidad, velocidad y vigor. La correlación obtenida entre el perímetro torácico y el peso, fue mayor a la descrita por Carroll 
y Huntington (1988) de 0,87 y a lo encontrado por Takaendegan et al. (2012) de 0,95 en animales de una raza local de Indonesia (Minahasa). Al correlacionar el peso y la longitud de los animales se obtuvo un valor de 0,93, dato superior a lo señalado por Carroll y Huntington (1988) de 0,75 , pero menor al informado por Takaendegan et al. (2012) de 0,96. Por último, la correlación entre perímetro torácico y longitud obtenida en este trabajo fue 0,92, valor mayor al obtenido por Takaendegan et al. (2012) de 0,56. A pesar de estas diferencias entre trabajos, las cuales se podrían relacionar al tipo de animal evaluado, las razas de las cuales descienden, a la interacción con el ambiente, al método empleado para la obtención de los datos y hasta la experiencia de los responsables en la toma de las medidas, los resultados obtenidos concuerdan en que las relaciones entre medidas corporales y el peso de los caballos presentaron valores positivos y se consideran correlaciones de moderadas a altas.

\section{Conclusiones}

Los resultados sugieren que las ecuaciones disponibles en la literatura presentaron diferencias en la estimación del peso del animal, según su etapa fisiológica, las cuales, promovieron en la mayoría de los casos una sobreestimación del peso, lo que podría provocar en los sistemas productivos, una mayor aplicación de productos veterinarios y un mayor aporte de nutrimentos a los animales que los requeridos por medio de la dieta ofrecida, debido a que estas prácticas utilizan de referencia el peso. Situación que se podría evitar con el uso de las ecuaciones desarrolladas en este trabajo para estimar el peso de caballos iberoamericanos, donde los productores tienen dos opciones para su cálculo, lo que permitirá un manejo apropiado, según la edad del animal, en aspectos nutricionales, sanitarios y de manejo en general, de acuerdo con la información que presente el sistema de producción sobre cada semoviente.

\section{Agradecimientos}

Se agradece a la Asociación Centroamericana de Criadores de Caballos de Raza Iberoamericana (ASOIBERO) por el apoyo y facilidades brindadas para esta investigación. Así también a los criadores, jaladores y montadores que brindaron la ayuda para el manejo de los animales.

\section{Literatura citada}

Almeida, M.R. 2010. Caracterización zoométrica y diagnóstico de los sistemas de producción de caballos mestizos de vaquería en el cantón de Rumiñahui. Tesis Lic., Escuela Superior Politécnica de Chimborazo, ECU.

ASOIBERO (Asociación Centroamericana de Criaderos de Caballos de Raza Iberoamericana). 2005. Reglamento para el registro genealógico del caballode raza Iberoamericana. ASOIBERO, San José, CRI.

ASOIBERO (Asociación Centroamericana de Criaderos de Caballos de Raza Iberoamericana). 2013. Patrón racial del caballo Iberoamericano. ASOIBERO, San José, CRI.

ASOIBERO (Asociación Centroamericana de Criadores de Caballos de Raza Iberoamericana). 2016. Registro genealógico. ASOIBERO, CRI. http://www.caballoibero.com/index.php/registro-genealogico (consultado 15 nov. 2016).

Carroll, C.L., and P.J. Huntington. 1988. Body condition scoring and weight estimation of horses. Equine Vet. J. 20:41-45. doi:10.1111/j.2042-3306.1988.tb01451.x

Cumming, B. 2009. Estimating a horse's condition and weight. Primefacts 928:1-12.

Gandarillas, M. 2008. Desórdenes ortopédicos en equinos: el factor nutricional. Agron. Forest. 35:30-33. 
Gamboa, J.B. 2012. Zoometría equina en el Trópico Húmedo de Costa Rica. Tesis Lic., EARTH, Guácimo, Limón, CRI.

García, N., A. Pérez, y G. Perrone. 2009. Estimación de peso corporal del caballo criollo mediante medidas morfométricas: Validación de ecuaciones publicadas para otras razas y desarrollo de nueva fórmula. REDVET 10(9). http://www. veterinaria.org/revistas/redvet/n090909/090906.pdf (consultado 15 nov. 2016).

Gibbs, P.G., and D.D. Householder. sf. Estimating horse body weight with a simple formula. HRG-012. Texas A\&M University, TX, USA. http://aglifesciences.tamu.edu/animalscience/wp-content/uploads/sites/14/2012/04/equine-estimating-horsebody-weight5.pdf (accessed Nov.15, 2016).

Gibbs, P.G., and G.D. Potter. SF. Feeding young horses for sound development. HGR-009. Texas A\&M University, TX, USA. https://aglifesciences.tamu.edu/animalscience/wp-content/uploads/sites/14/2012/04/nutrition-feeding-young-horses.pdf (accessed Nov. 15, 2016)

INEC (Instituto Nacional de Estadística y Censos). 2015. VI censo nacional agropecuario. INEC, San José, CRI.

Jones, R.S., T.L. Lawrence, A. Veevers, N. Cleave, and J. Hall. 1989. Accuracy of prediction of the live weight of horses from body measurements. Vet. Rec. 125:549-553. doi:10.1136/vr.125.22.549

Larrea, C.O. 2005. Caracterización zoométrica y diagnóstico de los sistemas de producción de caballos criollos en el cantón Chambo. Tesis Lic., Escuela Superior Politécnica de Chimborazo, ECU.

Larrea, C.O. 2014. Caracterización zoométrica y genética del caballo autóctono de los cantones Chambo y Guamote de la provincia de Chimborazo. Tesis MSc., Escuela Superior Politécnica de Chimborazo, ECU.

LaVigne, E.K., A.K. Jones, A.S. Londoño, A.S. Schauer, D.F. Patterson, J.A. Nadeau, and S.A. Reed. 2015. Muscle growth in young horses: effects of age, cytokines and growth factors. J. Anim. Sci. 93:5672-5680. doi:10.2527/jas.2015-9634

León, P. 2007. Manejo y cuidado del caballo. Escuela Universitaria de Ingeniería Técnica Agrícola, ESP.

Librado, P., A. Fages, C. Gaunitz, M. Leonardi, S. Wagner, N. Khan, K. Hanghøj, S.A. Alquraishi, A.H. Alfarhan, K. Al-Rasheid, C. Der-Sarkissian, M. Schubert, and L. Orlando. 2016. The evolutionary origin and genetic makeup of domestic horse. Genetics 204:423-434. doi:10.1534/genetics.116.194860

Martison, K.L., R.C. Coleman, A.K. Rendahl, Z. Fang, and M.E. McCue. 2014. Estimation of body weight and development of a body weight score for adult equids using morphometric measurements. J. Anim. Sci. 92:2230-2238. doi:10.2527/ jas.2013-6689

McGowan, C.M., L. Goff, and N. Stubbs. 2007. Animal physiotherapy: Assessment, treatment and rehabilitation of animals. Blackwell Publishing, NJ, USA.

McKiernan, B. 2007. Estimating a horse's weight. Primefacts 494:1-3.

Nieves, A., y F.C. Domínguez. 2009. Probabilidad y estadística para ingeniería: un enfoque moderno. McGraw Hill Interamericana, MEX.

Milner, J., and D. Hewitt. 1969. Weight of horses: Improved estimates base and the girth and length. Can. Vet. J. 10:314-316.

Pearson, N. 2007. Condition scoring and weight estimation of horses. Agriculture Notes AG0928. Horse Safety Australia, AUS. http://www.horsesafetyaustralia.com.au/wp-content/uploads/2010/09/Condition_Scoring_and_Weight_Estimation_of_ Horses.pdf. (accessed Jan. 1, 2017).

Rodríguez, J.A. 2016. Los orígenes de la raza. ASOIBERO. http://www.caballoibero.com/index.php/origenes-de-laraza?showall $=($ consultado 8 abr. 2019).

Solano, G., y R. WingChing-Jones. 2018. Estructura de hato y manejo nutircional, sanitario, reproductivo y ambiental del caballo iberoamericano en Costa Rica. Cuadernos Invest. 10:428-434. 
SAS Institute. 2012. SAS/STAT® 9.4 User's Guide. SAS Institute Inc., Cary, NC, USA.

Takaendegan, B.J., U. Paputungan, R.R. Noor, and S. Adiani. 2012. Live weight estimation by chest girth, body length and body volume formula in Minahasa local horse. Media Peternakan 35(2):80-84. doi:10.5398/medpet.2012.35.2.80 